\title{
Effects of Duplicity on the Period Changes of Cepheids
}

\section{SZABADOS}

Konkoly Observatory of the Hungarian Academy of Sciences, H-1525 Budapest, XII. P.O. Box 67, Hungary

\begin{abstract}
The light-time effect and the phase jump in the pulsation period of Cepheids as two characteristic manifestations of duplicity are discussed.
\end{abstract}

The frequency of known binaries among Cepheid variables has been continuously increasing. The first major study aimed at determining the frequency of Cepheid binaries was performed by Lloyd Evans (1968). He concluded that at least 15 per cent of Cepheids belong to spectroscopic binary systems of long period. The presence of the companion, however, has an influence on the photometric behavior of the variable star (see, e.g., Szabados, 1982; Coulson \& Caldwell, 1989), and the inclusion of various pieces of evidence resulted in revealing an increased percentage of Cepheid binaries: Burki (1984) determined a rate of binaries among Cepheids as high as 25-35\%. Both the use of the IUE satellite for detecting blue companions to Cepheids (Evans, 1992) and the numerous accurate radial velocity studies performed during the last decade lead to the conclusion that duplicity among Cepheids is a common feature. In a companion paper (Szabados 1992) evidence is put forward that more than fifty per cent of Cepheids belong to binary (or multiple) systems and, due to the strong observational selection effect a huge number of companions has not been pointed out yet.

It is a well known fact that the pulsation period of a regularly pulsating star can be determined much more accurately than any other stellar parameter. In the case of Cepheids, the relative error of the period determination is smaller than $10^{-5}$. This accuracy can only be achieved if the observations of the given singly periodic variable star are spread over a sufficiently long time interval and the pulsation period itself does not vary considerably. Fortunately a number of Cepheids meets these requirements, therefore these variables are suitable targets for the study of period changes using the $O-C$ diagram.

The results on the observed evolutionary period changes of Cepheids have been summarized by Szabados (1983). Period changes due to stellar evolution frequently occur in Cepheids, especially in the long period ones (which evolve faster than their shorter period and lower mass counterparts). As a Cepheid crosses the instability strip, its pulsation period is continuously increasing if the star evolves to the red in the HR-diagram, while the blueward passage through the instability strip causes continuous period decrease. The evolutionary period change shows up in the $O-C$ diagram as a parabola.

A companion to the Cepheid may cause a light-time effect due to the orbital motion. This kind of period change can be easily distinguished from the evolutionary effect. This long-period modulation in the pulsation period can be pointed out in the $O-C$ diagram of binary Cepheids of long period because in the case of short orbital periods the amplitude of the modulation is too low to be 
detectable. The other obvious limiting factor for generating a light-time effect is the orbital inclination. The situation is the same as for the spectroscopic binaries. Binaries with $\sin \mathrm{i} \ll 1$ are not suitable for searching for the orbital effect in these ways. A light-time effect, however, could be found in very few cases, the only Cepheid with spectroscopic confirmation being AW Per (Szabados 1991). The light-time effect interpretation of the $O-C$ diagram of FN Aql and RX Aur (Szabados 1988) still awaits confirmation. The other candidates possibly showing this phenomenon are those Cepheids whose spectroscopic-binary nature has been revealed and their $O-C$ diagrams show apparent period variations concordant with the expected orbital motion (e.g., AX Cir - Szabados 1989). Far more cases deserve much closer attention. The importance of the light-time effect lies in the fact that regular coverage of an $O-C$ diagram with data points needs only good quality photometric observations and this can be achieved much easier than to make regular spectroscopic studies. Of course, accuracy of the spectroscopically determined orbital parameters is much better, therefore the light-time effect discovered in Cepheid binaries can only be used for the rough determination of the orbital elements.

It has to be noted that the light-time effect has been discovered in some other types of pulsating variables, as well. Shorter pulsation periods favor the discovery of this subtle effect. The $O-C$ diagrams of the dwarf Cepheid SZ Lyn (Paparó et al. , 1988) and $\beta$ Cephei (the prototype of blue pulsators) (Pigulski \& Boratyn 1992) are very excellent examples for this phenomenon.

The other kind of period change characteristic of binary Cepheids is the phase jump seen in the $O-C$ diagram. These jumps can be either sudden or slower ("phase slip"), in this latter case an intermediate period is discernible before the pulsation of the star returns to the period valid previously. The phase jump is a very subtle effect but it seems to be very common among binary Cepheids: such behaviour has been discovered in more than 20 cases (see Szabados 1989, 1991).

The exclusive occurrence of the phase jump in binary Cepheids is based on solely empirical data. Each Cepheid showing a phase jump is either a member in a known spectroscopic binary or, suspected to have a companion on various grounds. Conversely, none of the well-studied single Cepheids shows this phenomenon. Nevertheless, a theoretical explanation would be desirable. The following brief description of facts connected with the phase jump will hopefully stimulate such model calculations.

The amount of the phase jump is usually several hundredth of the pulsation period. In most cases this phenomenon has been observed only once (e.g., SU Cyg, CV Mon) but there are several Cepheids showing more than one jump during the observed history of the star (e.g., X Lac, SZ Tau). Y Oph can be a key object in this respect. Its $O-C$ diagram (see Figure 1) implies that the occurrence of the phase jump is governed by the orbital motion because the cyclic appearance of the jumps has a cycle length identical with the orbital period (or its multiples). If this pattern of the $O-C$ graph keeps on showing this feature, then an obvious cause of the phenomenon can be the perturbing effect of the companion which exerts increased influence on the pulsating atmosphere of the Cepheid during the periastron passage. This offers a natural explanation for the synchronisation of the orbital motion and the phase jump. 


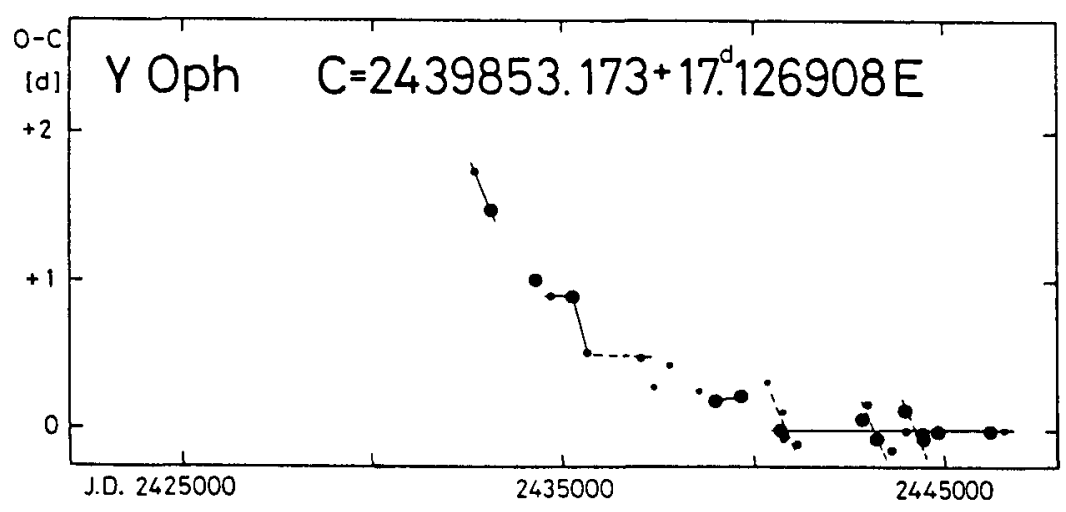

FIGURE 1. Multiple phase jumps in the $O-C$ diagram of $Y$ Ophiuchi.

Another intriguing fact is that the phase shift can alter the pulsational behavior of the Cepheid. The $O-C$ diagrams constructed for the maximum brightness of SU Cyg show a significantly smaller value of phase jump than for the median brightness (on the ascending branch of the light curve). This means that the ascending branch has become steeper since the "moment" of the phase jump of this particular Cepheid (see Figure 2).

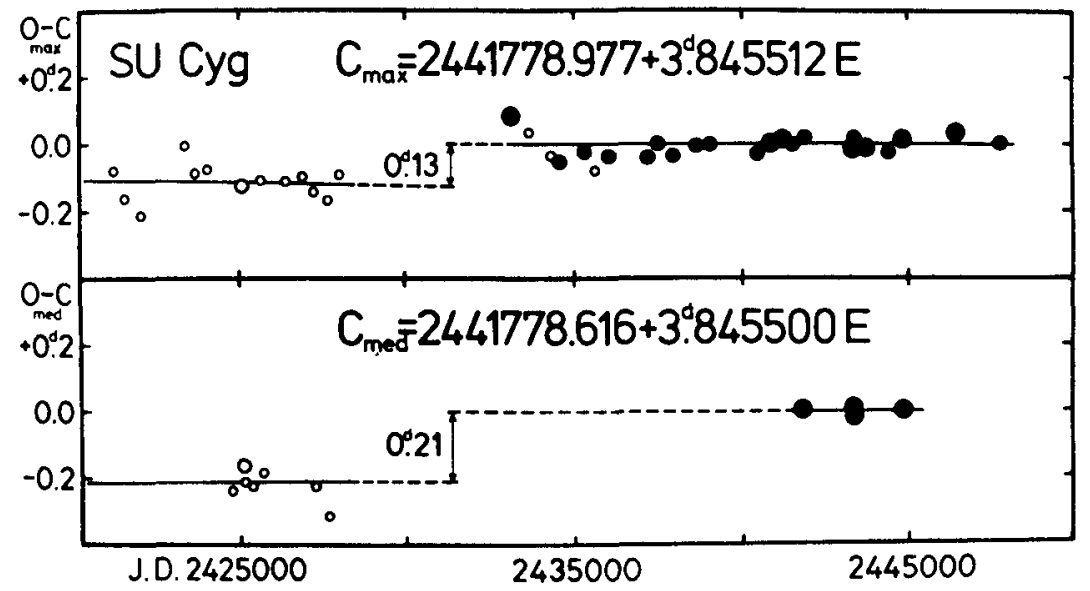

FIGURE 2. The phase jump in the $O-C$ diagram of SU Cygni. The different values for the jump of maximum and median brightnesses indicate that the shape of the light curve has also changed. 
It is important to emphasize that the distinction between the light-time effect and the phase jump is not always easy if the number of the $O-C$ residuals is scanty and radial velocity data are not available. The phase jump in the $O-C$ diagram, however, is undoubtedly observed. The most striking proof of its existence seems to be the case of RS Ori where the phase jump and the light-time effect co-exist in the same $O-C$ diagram. Nevertheless, further observations of this Cepheid would be desirable.

The stepwise structure of the $O-C$ graph as a result of the phase jump occurring in Cepheids belonging to binary systems is in contradiction with the current evolutionary theory which predicts a continuous variation in the pulsation period, i.e. a parabolic $O-C$ graph. Thus the presence of the companion may affect the evolution of the Cepheid component. This behavior also deserves a careful theoretical study.

The author is indebted to the Organizing Committees of the Colloquium, the International Astronomical Union, and the Hungarian Soros Foundation for the grants that made his participation in the Colloquium possible.

\section{REFERENCES}

Burki, G. 1984, $A \& A, 133,185$

Coulson, I.M. \& Caldwell, J.A.R. 1989, MNRAS, 240, 285

Evans, N.R. 1992, ApJ, 384, 233

Lloyd Evans, T. 1968, MNRAS, 141, 109

Paparó, M., Szeidl, B., \& Hamdy, M.A. 1988, Ap\&SS, 149, 73

Pigulski, A. \& Boratyn, D.A. 1992, $A \& A, 253,178$

Szabados, L. 1982, Comm. Konkoly Obs. Hung. Acad. Sci., Budapest, No.83, 209

Szabados, L. 1983, ApESS, 96, 185

Szabados, L. 1988, PASP, 100, 589

Szabados, L. 1989, Comm. Konkoly Obs. Hung. Acad. Sci., Budapest, No. 94

Szabados, L. 1991, ibid. No. 96

Szabados, L. 1992, this Colloquium 